POS $\quad$ PROCEEDINGS

\title{
On the phase of quark determinant in lattice QCD with finite chemical potential
}

\author{
Shinji Takeda ${ }^{* a, b}$, Yoshinobu Kuramashi $^{b, c, d}$ and Akira Ukawa ${ }^{d}$ \\ ${ }^{a}$ Institute of Physics, Kanazawa University, Kanazawa 920-1192, Japan \\ ${ }^{b}$ RIKEN Advanced Institute for Computational Science, Kobe, Hyogo 650-0047, Japan \\ ${ }^{c}$ Graduate School of Pure and Applied Sciences, University of Tsukuba, Tsukuba, Ibaraki \\ 305-8571, Japan \\ ${ }^{d}$ Center for Computational Sciences, University of Tsukuba, Tsukuba, Ibaraki 305-8577, Japan
}

The XXIX International Symposium on Lattice Field Theory - Lattice 2011

July 10-16, 2011

Squaw Valley, Lake Tahoe, California

\footnotetext{
* Speaker.

${ }^{\dagger}$ E-mail: takeda@hep.s.kanazawa-u.ac.jp
} 


\section{Introduction}

Understanding the property of the phase of quark determinant is crucial to control the sign problem in lattice QCD with finite chemical potential. So far the phase has been studied mainly by two approaches: The first one is on the lattice [1, 2, 3, 4] and there, the Taylor expansion is used to calculate the phase. Its convergence range, however, is rather restricted to a narrow region, $\mu / T<1$. The other approach is the effective theory or the random matrix model [5, 6, 7, 8]. Near the phase transition, the low energy effective theories may not be reliable. To survey such a region, it is imperative to work within the first principle framework of QCD itself.

In this context, our motivation here is to obtain and understand the phase of the determinant more precisely, and irrespective of the location of the phase diagram. Especially, we are interested in the low $T$ and high $\mu$ region. Some details and definition of undefined quantity are given in 9 .

\section{Winding number expansion (WNE)}

A key idea for the purpose given above is the winding number expansion. Actually, this has good convergence property. To see this, first of all, let us consider the determinant of the WilsonDirac operator, and as well known this can be expressed by the winding number expansion,

$$
\operatorname{det}[D(\kappa, \mu)]=A_{0}(\kappa) \exp \left[-\sum_{q \in \mathbb{Z}} e^{q \mu / T} V^{(q)}(\kappa)\right] .
$$

Note that this is a fugacity expansion of the logarithm of determinant. $A_{0}$ is independent of $\mu$ and in the following we only consider the essential part of the phase, namely the exponential factor. Here $q$ is the winding number and $V^{(q)}$ is a sum of all gauge loops which wind around the timedirection $q$-times, and is a trace of the $\mathrm{SU}(3)$ matrix, so this is just a complex number. This has a relationship $V^{(-q)}=V^{(q) *}$. The relationship means that the clockwise loop and anti-clockwise loop are related with each other by complex conjugate. After some rearrangement with this formula, we can identify the pure imaginary part of the exponent as the phase,

$$
\theta(\mu)=\arg \left(A_{0}\right)-\sum_{q=1}^{\infty} 2 \sinh (q \mu / T) \operatorname{Im}\left[V^{(q)}\right] .
$$

Here let us roughly estimate the convergence range of this expansion. If we look at $V^{(q)}$ which is consisted of the link variables, the each link variable comes together with the hopping parameter $2 \kappa$. Therefore a longer loop is strongly suppressed for a typical value $\kappa=0.125$. For example, $V^{(q)}$ whose length is minimally $N_{\mathrm{T}}$ times $q$, is of order $\kappa^{q N_{\mathrm{T}}}$, and this is very strong suppression. So now it is a competition between the small factor $V^{(q)}$ and large factor $\sinh (q \mu / T)$. A competition between them determines the convergence range. An effective expansion parameter now is $\left\{e^{\mu / T+N_{\mathrm{T}} \ln (2 \kappa)}\right\}^{q}$ for large $q$. This expansion will converge if the exponent here is negative $\mu / T<-N_{\mathrm{T}} \ln (2 \kappa)$. The convergence range for a typical value of $\kappa=0.125$ which gives $\ln (0.125)=-1.386$.. is much wider than the Taylor expansion.

To check such behavior, we did some simulation whose parameters are the same as that of Kentucky group [11] $a=0.328 \mathrm{fm}, m_{\pi}=834 \mathrm{MeV}$, with lattice size $N_{\mathrm{L}}=6$ and $N_{\mathrm{T}}=4,6,8,12$. 

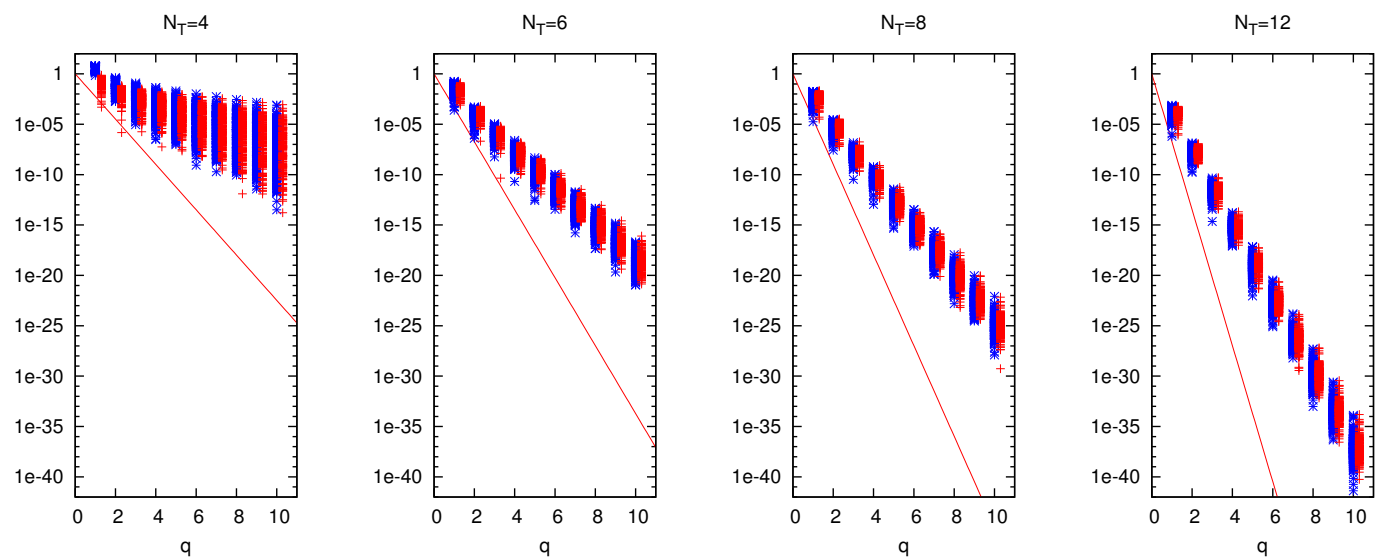

Figure 1: Absolute values of real (blue points) and imaginary (red points) parts of $V^{(q)}$ for $q=1,2, \ldots, 10$ with various $N_{\mathrm{T}}$. They are measured at $a \mu=0.2$ employing 200 phase-quenched configurations. Red line denotes an expected asymptotic behavior $(2 \kappa)^{q N_{\mathrm{T}}}$ as written in the text.
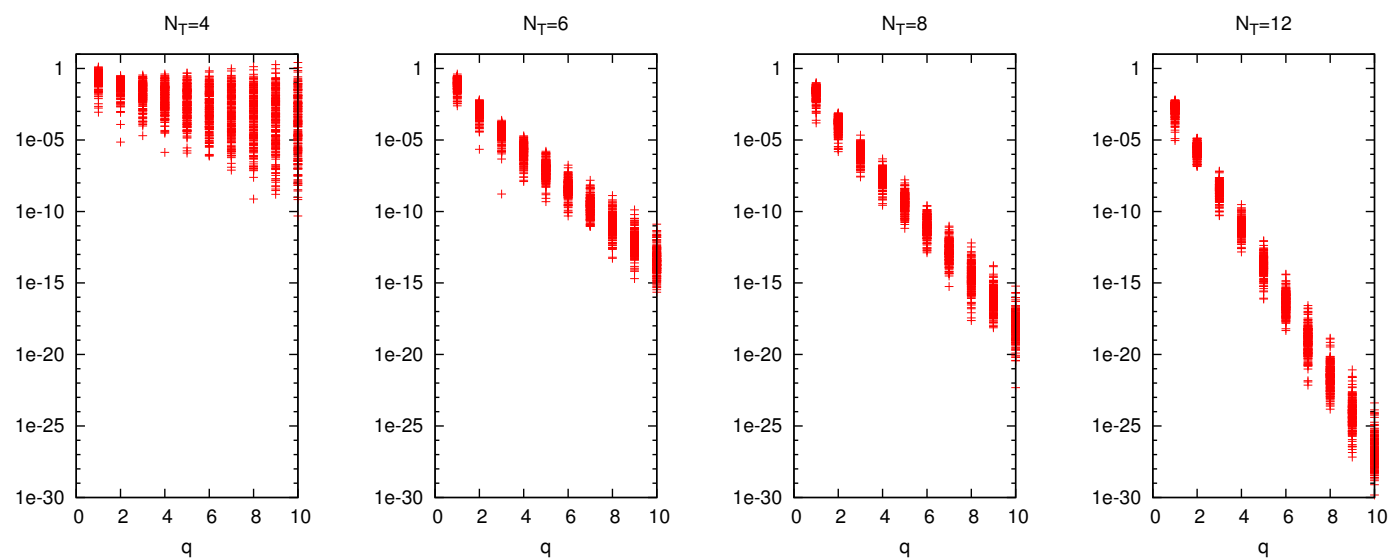

Figure 2: Absolute value of each term of the phase: $2 \sinh (q \mu / T)\left|\operatorname{Im}\left[V^{(q)}\right]\right|$ for $q=1,2, \ldots, 10$ with various $N_{\mathrm{T}}$ on the same configurations as before

With these input parameters, we generate 200 phase quenched configurations and then measure the terms in eq.2.2) from $q=1$ to 10 . Here $a \mu$ is fixed to 0.20 , but $N_{\mathrm{T}}$ is changed from 46 to 8 . We can see that $N_{\mathrm{T}}=4$ result does not converge but for larger $N_{\mathrm{T}}$ the convergence is getting better. So we could confirm the nice convergent behavior numerically.

\section{Upper bound for the phase}

Let us derive an upper bound of the phase. We begin with the inequality $|x+y| \leq|x|+|y|$ for $x, y \in \mathbb{C}$ repeatedly to find that

$$
|\theta| \leq \sum_{q=1}^{\infty} 2 \sinh (q \mu / T)\left|\operatorname{Im}\left[V^{(q)}\right]\right| \leq \sum_{q=1}^{\infty} 2 \sinh (q \mu / T)\left|V^{(q)}\right| .
$$



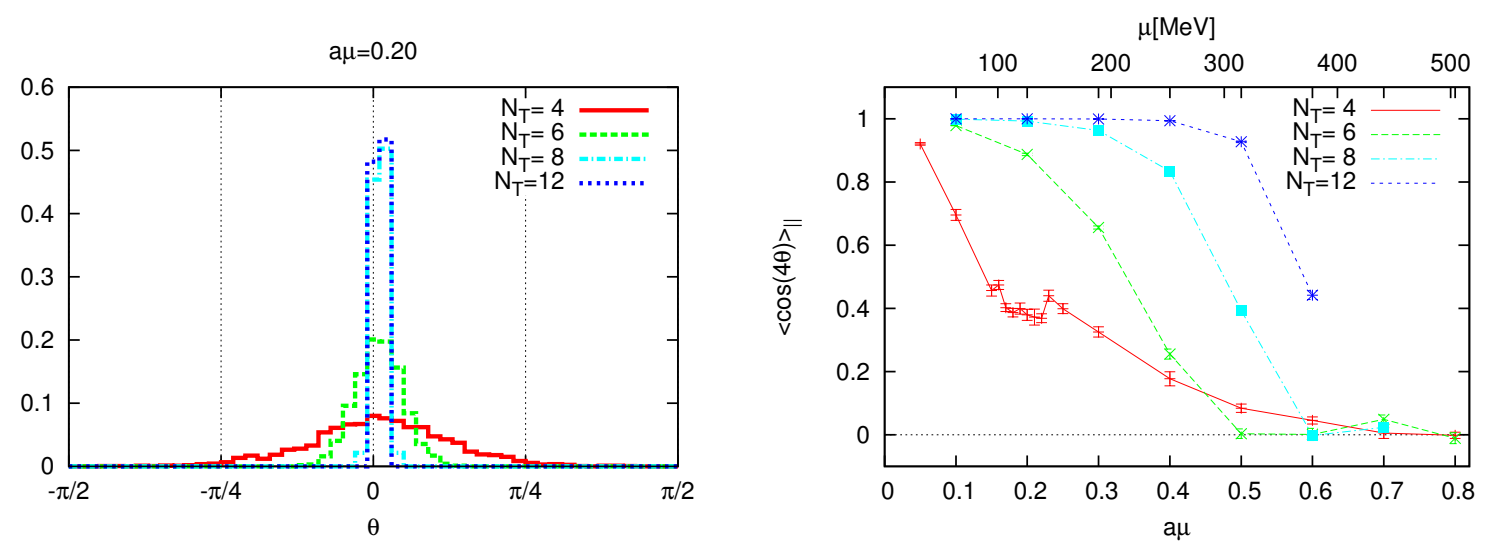

Figure 3: Left panel:Histogram of the phase at $a \mu=0.2$ with various $N_{\mathrm{T}}$. Other parameters are the same as those of the previous figure. Right panel:Averaged reweighting factor as as function of $a \mu$ with various $N_{\mathrm{T}}$.

Next step is to truncate the winding number expansion at $q=1$. After this truncation, the upper bound becomes

$$
\sum_{q=1}^{\infty} 2 \sinh (q \mu / T)\left|V^{(q)}\right| \longrightarrow 2 \sinh (\mu / T)\left|V^{(1)}\right|
$$

And then we apply the hopping parameter expansion to the $V^{(1)}$ and then the leading term turns out to be this form.

$$
\begin{aligned}
\left|V^{(1)}\right| & =\left|\operatorname{tr}\left(H_{+}\right)\right|+O\left((2 \kappa)^{2 N_{\mathrm{T}}}\right) \\
& =(2 \kappa)^{N_{\mathrm{T}}} \cdot \operatorname{tr}_{\text {spin }}\left[\left(P^{(4)}\right)^{N_{\mathrm{T}}}\right] \cdot\left|\sum_{\mathbf{x}} \operatorname{tr}_{\text {color }}\left[\prod_{x_{4}=1}^{N_{\mathrm{T}}} U_{4}\left(\mathbf{x}, x_{4}\right)\right]\right|+O\left(N_{\mathrm{T}}(2 \kappa)^{N_{\mathrm{T}}+2}\right) \\
& \leq(2 \kappa)^{N_{\mathrm{T}}} \cdot 2 \cdot 3 N_{\mathrm{L}}^{3},
\end{aligned}
$$

where $P+$ is the projection operator for the time direction. We have used the fact that the polyakov loop is just a trace of $\mathrm{SU}(3)$ matrix thus this has an upper bound $3 N_{\mathrm{L}}^{3}$. By combining all, one can arrive at an approximated upper bound for the phase.

$$
|\theta| \leq 12 N_{\mathrm{L}}^{3}(2 \kappa)^{N_{\mathrm{T}}} \sinh (\mu / T) .
$$

This says that the phase grow with the spatial volume factor but gets exponentially suppressed by increasing $N_{\mathrm{T}}$. So in a finite box and large $N_{\mathrm{T}}$, one can set an upper bound.

To examine the behavior of phase, we measure the phase exactly, that is, no noise, no expansion, on the same phase quenched configurations as before and its histogram is shown in the left panel of Fig. 3. The smaller $N_{\mathrm{T}}$ cases show wide distribution, but for large $N_{\mathrm{T}}$ it is getting sharp. So we confirmed that the fluctuation of the phase gets small for large $N_{\mathrm{T}}$.

The above result indicates that for large $N_{\mathrm{T}}$, the reweighting factor is very close to unity. So, we measure it with the various value of $a \mu$ as shown in the right panel of Fig. 3 . Note that we fix the lattice spacing. So changing the $N_{\mathrm{T}}$ means changing the temperature. The small $N_{\mathrm{T}}$ data takes rather small value, but for large $N_{\mathrm{T}}$, it tends to get close to the unity. 

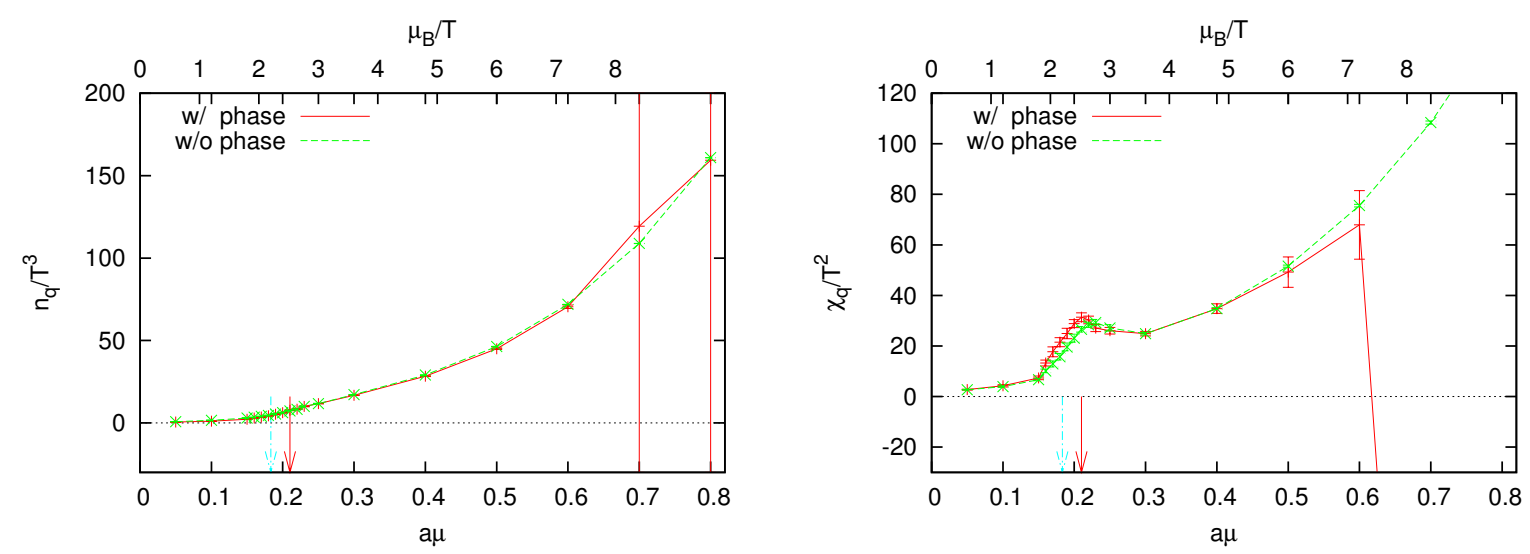

Figure 4: Quark number density (upper) and its susceptibility (lower) as a function of $a \mu$ (or the baryon chemical potential $\mu_{\mathrm{B}}=3 \mu$ in unit of the temperature for upper horizontal axis) with and without the phase factor. Light blue dotted arrow shows the location of transition point determined by the canonical approach [11. Red solid arrow shows the location of a peak of the quark number susceptibility $(a \mu=0.21)$. The results at $a \mu=0.7$ and 0.8 have the huge error bar because of the extremely small reweighting factor (an appearance of the sign problem) as shown in the previous figure.

\section{Phase reweighting simulation}

From this nice behavior of the reweighting factor, we got a motivation to do the phase reweighting.

$$
\langle\mathscr{O}\rangle=\frac{\left\langle\mathscr{O} \mathrm{e}^{i N_{\mathrm{f}} \theta}\right\rangle_{\|}}{\left\langle\mathrm{e}^{i N_{\mathrm{f}} \theta}\right\rangle_{\|}}
$$

where the reweighted and the phase-quenched ensembles averages are defined as

$$
\begin{aligned}
&\langle\mathscr{O}\rangle=\frac{\int[d U] \mathrm{e}^{-S_{\mathrm{G}}}(\operatorname{det} D)^{N_{\mathrm{f}}} \mathscr{O}[U]}{\int[d U] \mathrm{e}^{-S_{\mathrm{G}}}(\operatorname{det} D)^{N_{\mathrm{f}}}}, \\
&\langle\mathscr{O}\rangle_{\|}=\frac{\int[d U] \mathrm{e}^{-S_{\mathrm{G}}}|\operatorname{det} D|^{N_{\mathrm{f}}} \mathscr{O}[U]}{\int[d U] \mathrm{e}^{-S_{\mathrm{G}}}|\operatorname{det} D|^{N_{\mathrm{f}}}} .
\end{aligned}
$$

Before showing the results, we want to give a remark that we used a reduction technique, and thanks to that we can now exactly calculate the phase, the quark Number and its susceptibility. So, from now forget about the winding number expansion.

Fig. 4 is the number density and susceptibility together with the phase quenched results. For larger $a \mu$, the data is noisy due to the small reweighting factor as shown before. The light blue arrow indicates the transition point at $150 \mathrm{MeV}$ which is determined by the canonical approach by Kentucky group [11]. Such transition like behavior can be clearly seen in the Susceptibility where a peak is observed and its location (the red arrow) is roughly consistent with that of Kentucky group.

Such transition like behavior can also be observed in the polyakov loop in Fig. 5. The location of the peak in its susceptibility is almost same as that of the quark number.

Fig. 6 is a histogram of the plaquette value on the phase-quenched configuration. $N_{\mathrm{T}}$ is fixed to 4 , and $a \mu$ is changed. We can see a double peak at $a \mu=0.22$ therefore this is very likely the 

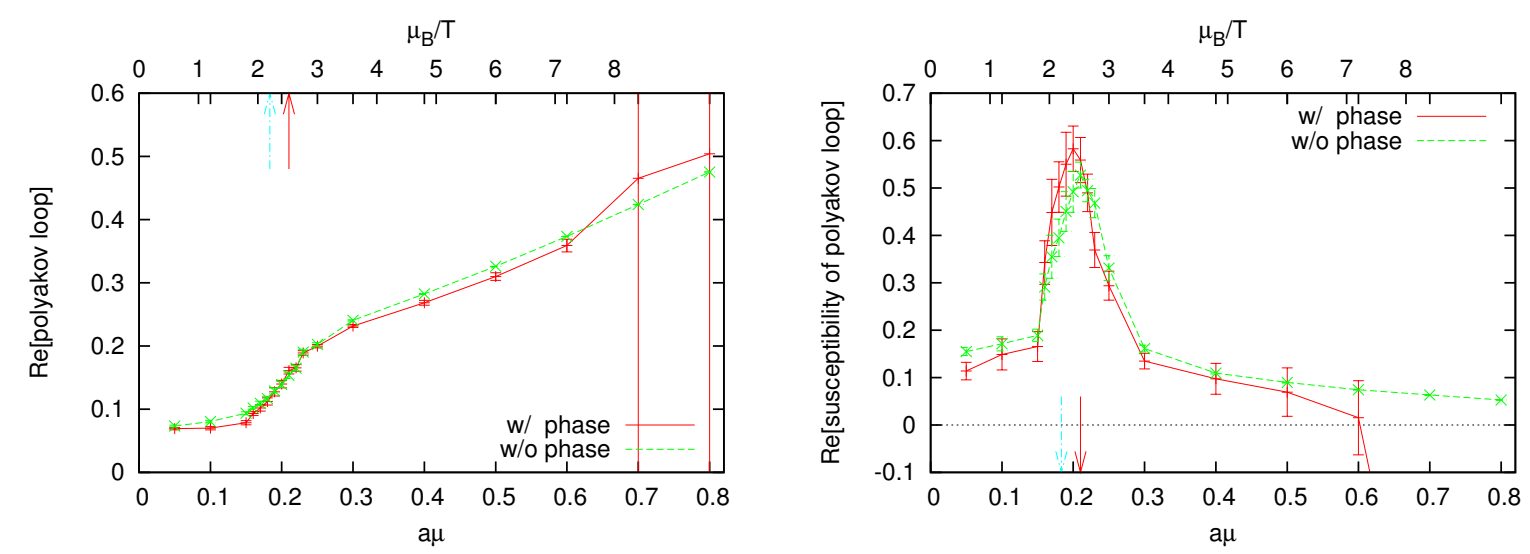

Figure 5: Averaged real part of the polyakov loop (upper) and its susceptibility (lower) as a function of $a \mu$ with and without the phase factor.
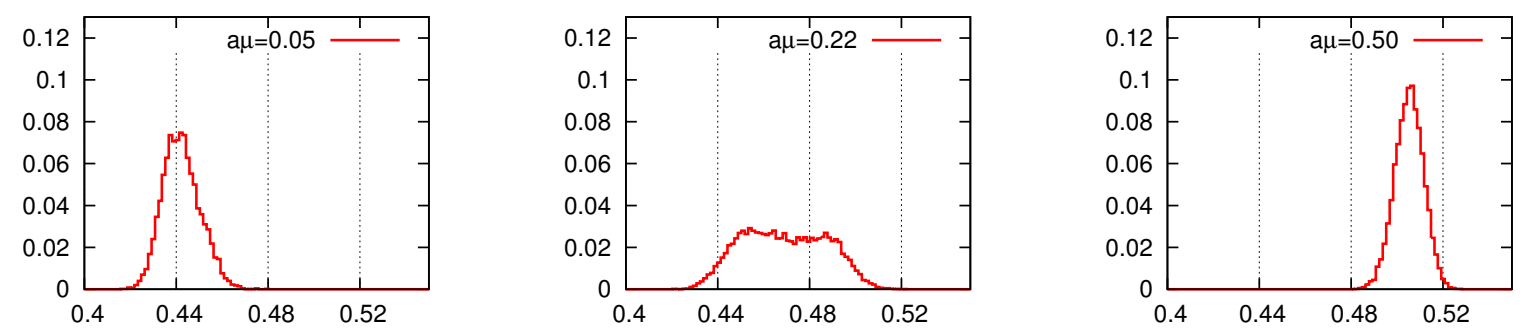

Figure 6: Histogram of plaquette value on the phase-quenched configuration for $N_{\mathrm{T}}=4$ with various $a \mu$.

first order phase transition. Of course, to pin down the order of the phase transition, we have to do a finite size scaling.

This work is supported in part by the Grants-in-Aid for Scientific Research from the Ministry of Education, Culture, Sports, Science and Technology (Nos. 23105707, 23740177, 22244018, 20105002). The numerical calculations have been done on T2K-Tsukuba and T2K-Tokyo cluster system at University of Tsukuba and University of Tokyo respectively.

\section{References}

[1] C. Allton et al., Phys.Rev. D66 (2002) 074507.

[2] Y. Sasai, A. Nakamura and T. Takaishi, Nucl.Phys.Proc.Suppl. 129 (2004) 539.

[3] S. Ejiri, Phys.Rev. D69 (2004) 094506.

[4] S. Ejiri, Phys.Rev. D73 (2006) 054502.

[5] K. Splittorff and J. Verbaarschot, Phys.Rev.Lett. 98 (2007) 031601.

[6] K. Splittorff and J. Verbaarschot, Phys.Rev. D75 (2007) 116003.

[7] K. Splittorff and J. Verbaarschot, Phys.Rev. D77 (2008) 014514. 
[8] J. Han and M. A. Stephanov, Phys. Rev. D78 (2008) 054507.

[9] S. Takeda, Y. Kuramashi and A. Ukawa, arXiv:1111.6363 [hep-lat].

[10] J. Danzer and C. Gattringer, Phys. Rev. D78 (2008) 114506.

[11] A. Li, A. Alexandru, K.-F. Liu and X. Meng, Phys.Rev. D82 (2010) 054502.

[12] D. T. Son and M. A. Stephanov, Phys. Rev. Lett. 86 (2001) 592. 